\title{
Effects of hydrogen adsorption on single-wall carbon nanotubes: Metallic hydrogen decoration
}

\author{
O. Gülseren, ${ }^{1,2}$ T. Yildirim, ${ }^{1}$ and S. Ciraci ${ }^{3}$ \\ ${ }^{1}$ NIST Center for Neutron Research, National Institute of Standards and Technology, Gaithersburg, Maryland 20899 \\ ${ }^{2}$ Department of Materials Science and Engineering, University of Pennsylvania, Philadelphia, Pennsylvania 19104 \\ ${ }^{3}$ Department of Physics, Bilkent University, Ankara 06533, Turkey
}

(Received 22 July 2002; published 6 September 2002)

\begin{abstract}
We show that the electronic and atomic structure of carbon nanotubes undergo dramatic changes with hydrogen chemisorption from first principle calculations. Upon uniform exohydrogenation at half coverage, the cross sections of zigzag nanotubes become literally square or rectangular, and they are metallic with very high density of states at the Fermi level, while other isomers can be insulating. For both zigzag and armchair nanotubes, hydrogenation of each carbon atom from inside and outside alternatively yield the most stable isomer with a very weak curvature dependence and a large band gap.
\end{abstract}

DOI: 10.1103/PhysRevB.66.121401

PACS number(s): 73.22.-f, 68.43.Bc, 68.43.Fg

Single wall carbon nanotubes (SWNTs) $)^{1,2}$ are among the most attractive systems for fabricating nanodevices because they exhibit many unusual mechanical and electronic properties. Variation in the chiral vector or a small radial deformation result in marked changes ranging from insulating to ideal one-dimensional (1D) conducting properties. ${ }^{3-7}$ The physical and chemical properties SWNTs can also be efficiently engineered by the adsorption of atoms or molecules on nanotubes..$^{8-15}$ Recently, it has been shown that the chemical activity of carbon nanotubes also depends on the chirality and radius, ${ }^{16,17}$ denoting tunable absorption of atoms on SWNTs by structural deformation. ${ }^{17,18}$ The interplay between adsorption and electromechanical properties can give rise to interesting physiochemical properties. ${ }^{17,18}$ The experimentally observed sensitivity of the electronic properties of SWNTs to the presence of oxygen and hydrogen is clear evidence for the importance of this interplay. ${ }^{19}$

Motivated by these considerations, we have investigated the structural and electronic properties of hydrogenated SWNTs (H-SWNT) as a function of hydrogen coverage and decoration (i.e. isomers) by extensive first principles calculations. Our results indicate that hydrogen adsorption on nanotubes gives rise to many properties which can mediate important applications in molecular electronics. One of our most important results is that upon hydrogenation at uniform half coverage, the zigzag $(n, 0)$ SWNTs are metallized with high density of states at the Fermi level and the circular cross sections of the tubes are changed to square or rectangle ones. The carbon atoms near the corners form new diamondlike $\mathrm{C}-\mathrm{C}$ bonds. Therefore, these carbon atoms are electronically and chemically passive, isolating the four conducting faces of the H-SWNT. Hence, loosely speaking a uniform half-coverage $(n, 0) \mathrm{H}$-SWNT is composed of four-wire nanocable.

Our study comprises zigzag $((7,0),(8,0),(9,0),(10,0)$, $(12,0))$ and armchair $((6,6),(10,10))$ SWNTs which are hydrogenated at two different coverages. For full coverage $(\Theta=1)$, we consider two isomers; namely exohydrogenation where each carbon atom is bonded to a hydrogen atom from outside of the nanotube (labeled by $\mathrm{C}_{4 n} \mathrm{H}_{4 n}$ ) and endoexohydrogenation where each carbon atom is bonded to a hydrogen from inside and outside of the tube alternatively (labeled by $\left.\mathrm{C}_{4 n} \mathrm{H}_{2 n} \mathrm{H}_{2 n}\right)$. For half coverage $(\Theta=0.5)$, we consider the three most interesting isomers (labeled by $\mathrm{C}_{4 n} \mathrm{H}_{2 n}$ ); namely (i) uniform pattern where every other carbon atom is bonded to a hydrogen from outside, (ii) chain pattern where every other carbon zigzag chain is saturated by hydrogen, and (iii) dimer pattern where every other carbon dimer rows perpendicular to the zigzag carbon chains are saturated by hydrogen. Fig. 1(a) shows these three isomers at half coverage.

The first principles total energy and electronic structure calculations have been carried out within the generalized gradient approximation $(\mathrm{GGA})^{20}$ using the pseudopotential plane wave method ${ }^{21}$ in a supercell geometry. Details of the parameters used in this work are the same with those given in Ref. 17. Fully relaxed geometries are obtained by optimizing all atomic positions and the lattice constant $c$ along the tube axis until the maximum force and stress are less than $0.01 e V / \AA$ and $0.1 \mathrm{GPa}$, respectively.

We find that geometric and electronic structures and binding energies of H-SWNTs strongly depend on the pattern of hydrogenation (i.e. decoration). The most remarkable effect is obtained when zigzag nanotubes are uniformly exohydrogenated at half coverage $(\Theta=0.5)$. Upon hydrogenation the structure undergoes a massive reconstruction, whereby circular cross section of the $(7,0)$ SWNT changes to a rectangular one, and those of $(8,0),(9,0),(10,0)$ and $(12,0)$ change to square ones as shown in Fig. 1(b). These new structures are stabilized by the formation of new diamondlike $\mathrm{C}-\mathrm{C}$ bonds with $d_{C C} \sim 1.51-163 \AA$ near the corners of rectangular or square H-SWNTs. Hence, triangular and pentagonal $\mathrm{C}$ rings are formed instead of hexagonal. Depending on $(2 n \bmod 4)$, either one bond is formed just at the corners or two bonds at either side of the corners. Most interestingly, all these structures are found to be metallic with a very large density of states at the Fermi level. The uniform adsorption at $\Theta=0.5$ for zigzag nanotubes are metastable. Such a local minimum does not exist for armchair nanotubes, since uniformly adsorbed $\mathrm{H}$ atoms are rearranged upon relaxation by concerted exchange of $\mathrm{C}-\mathrm{H}$ bonds to form zigzag chains along the tube axis. Several snap shots for hydrogen dimerization on an armchair tube are shown in Fig. 1(c). The cross sections 


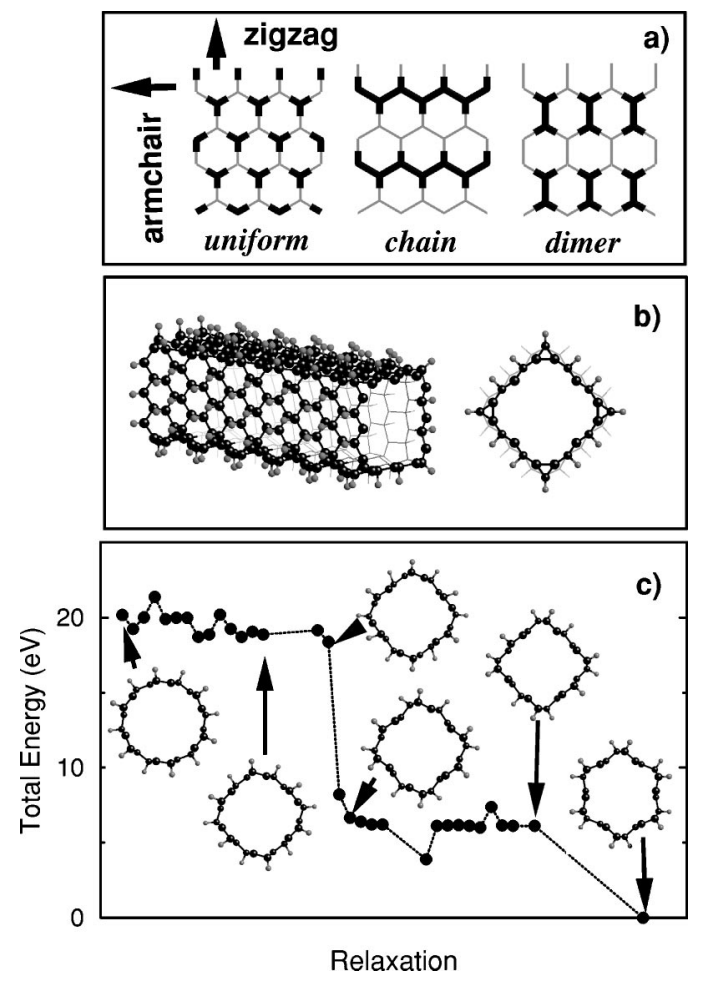

FIG. 1. (a) A view of three different isomers of H-SWNT at half coverage. The left and up arrows indicate the tube axis for armchair and zigzag nanotubes, respectively. Carbon atoms which are bonded to hydrogens are indicated by dark color. (b) A side and top view of a $(12,0) \mathrm{H}-\mathrm{SWNT}$, indicating the square cross section of a uniformly exohydrogenated nanotube at half coverage. (c) Several snap shots during the relaxation steps of an armchair $(6,6)$ $\mathrm{H}-\mathrm{SWNT}$, indicating that an uniform exohydrogenation at half coverage is not stable against forming a chain isomer.

of chain isomer at $\Theta=0.5$ of armchair tubes are polygonal where the corners are pinned by the zigzag $\mathrm{H}$ chains along the tube axis. For other isomers at half coverage as well as exo- and endo-exohydrogenations at full coverage, the cross sections remain quasicircular (see Fig. 2).

The average binding energies of $\mathrm{H}$ are obtained from the expression,

$$
E_{b}=\left(E_{T, C_{4 n}}+m E_{H}-E_{T, C_{4 n} H_{m}}\right) / m
$$

in terms of the total energies of the bare SWNT, $E_{T, C_{4 n}}$, the hydrogen covered SWNT, $E_{T, C_{4 n} H_{m}}$, and the energy of atomic hydrogen, $E_{H}$ ( $m$ is the number of $\mathrm{H}$ atoms per unit cell). According to the above definition, stable structures have positive binding energies. The average binding energies as a function of nanotube radius are shown in Fig. 2. The variation of $E_{b}$ with the radius of the bare nanotube can be fitted to a simple formula, ${ }^{16,17}$

$$
E_{b}=E_{o}(\Theta)+\frac{C_{p}(\Theta)}{R^{p}} .
$$

Note that $1 / R$ form (i.e. $p=1$ ) is quite common to SWNTs and scales various properties. ${ }^{4,5,7,16,17}$ Here $E_{o}(\Theta)$ is the

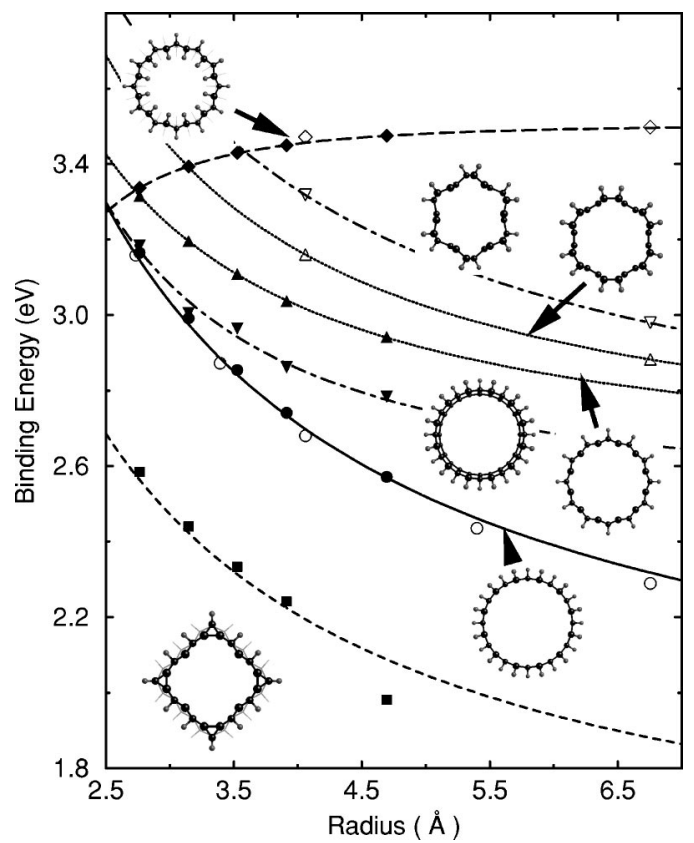

FIG. 2. Average binding energies, $E_{b}$, of hydrogen atoms adsorbed on various zigzag and armchair SWNTs versus bare tube radius $R$. Filled and open symbols are for zigzag and armchair nanotubes, respectively. Circles and diamonds are for exo- and endo-exohydrogenation at full coverage, respectively. The filled squares show the zigzag nanotubes uniformly exohydrogenated at half coverage. The chain and dimer patterns of adsorbed hydrogen atoms at half coverage are shown by down- and up-triangles, respectively. Curves are analytical fits explained in the text. Insets show top view of several H-SWNT isomers.

binding energy when $R \rightarrow \infty$, and hence corresponds to the adsorption of $\mathrm{H}$ on graphene at a given coverage, while $C_{p}(\Theta)$ is a constant that depends on coverage $\Theta$, and represents the curvature effect. Calculated $E_{b}$ 's are fitted to Eq. (2) with the values listed in Table I. While $E_{b}$ increases with decreasing $R$ in the case of exohydrogenation, this trend is reversed for endo-exohydrogenation due to increased $\mathrm{H}-\mathrm{H}$ repulsion inside the tube at small $R$. Nevertheless, the endoexohydrogenation of SWNTs, which transforms the $s p^{2}$ to $s p^{3}$-like bonding, gives rise to the highest binding energy saturating at $3.51 \mathrm{eV}$ as $R \rightarrow \infty$. At this limit, the exoendohydrogenated graphene (from above and below) is

TABLE I. Values of the parameters $E_{0}(\mathrm{eV})$ and $C_{p}(\Theta)$ ( $\mathrm{eV} \AA^{p}$ ) given in Eq. (2) to fit the binding energies.

\begin{tabular}{lccc}
\hline \hline $\begin{array}{l}\text { Hydrogen } \\
\text { decoration }\end{array}$ & $E_{0}$ & $\begin{array}{c}\text { Zigzag Tubes } \\
C_{p}(\Theta)\end{array}$ & $\begin{array}{c}\text { Armchair Tubes } \\
C_{p}(\Theta)\end{array}$ \\
\hline Exo $\Theta=1.0$ & 1.75 & $C_{1}=3.87$ & $C_{1}=3.87$ \\
Exo-endo $\Theta=1.0$ & 3.51 & $C_{3}=-3.49$ & $C_{3}=-3.49$ \\
Uniform $\Theta=0.5$ & 1.41 & $C_{1}=3.19$ & not stable \\
Chain $\Theta=0.5$ & 2.50 & $C_{1}=0.53$ & $\begin{array}{c}C_{1}=3.16 \\
\end{array}$ \\
& & $+C_{2}=3.70$ & $+C_{2}=0.73$ \\
Dimer $\Theta=0.5$ & 2.55 & $C_{1}=1.41$ & $C_{1}=1.87$ \\
& & $+C_{2}=1.94$ & $+C_{2}=2.42$ \\
\hline \hline
\end{tabular}




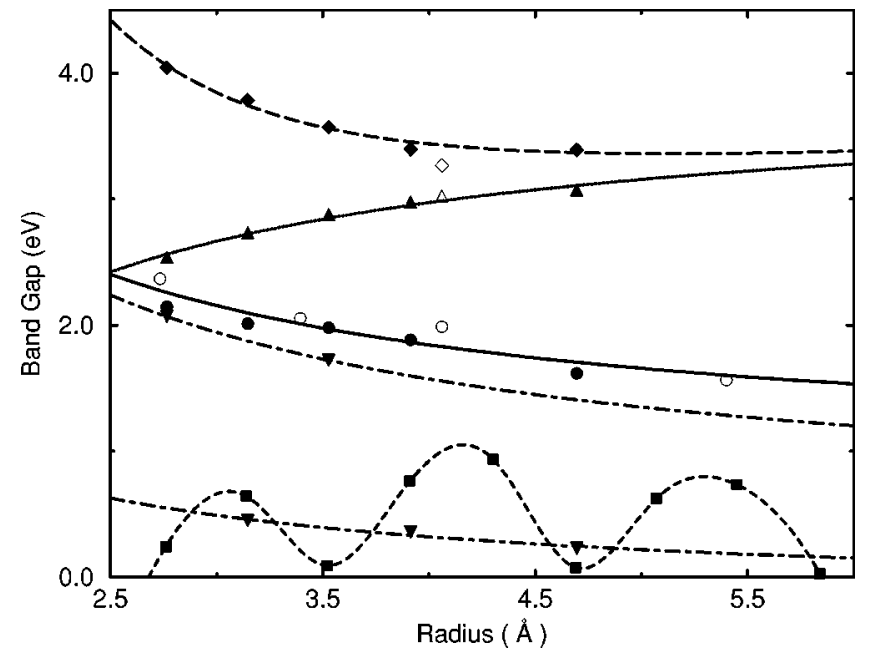

FIG. 3. The band gaps, $E_{g}$, versus the bare nanotube radius $R$. Filled and empty symbols indicate zigzag and armchair SWNTs, respectively. Squares show nonmonotonic variation of the band gap of the bare zigzag nanotubes. Exo and endo-exohydrogenated nanotubes $(\Theta=1)$ and chain and row patterns of adsorbed hydrogen atoms at half coverage are shown by circles, diamonds, down- and up-triangles, respectively. Lines are guide to eye.

buckled by $0.46 \AA$ as if two diamond (111) planes with interplanar distance of $0.50 \AA$.

As with the atomic structure, the electronic structure of SWNTs undergo important changes as a result of hydrogenation. For bare zigzag SWNTs the variation of the band gap, $E_{g}$, with $n$ is rather complex due to interplay between zone folding and curvature induced $\sigma^{*}-\pi^{*}$ mixing ${ }^{7,22}$ while bare armchair SWNTs are metallic. Here we find that sizable band gaps are opened as a result of hydrogenation (Fig. 3). At $\Theta$ $=1$, the band gap displays a similar behavior for both types of nanotubes and hydrogenation, and decreases with inreasing $R$. Relatively larger band gaps (in the range of $3.5-4 \mathrm{eV}$ ) of endo-exohydrogenated SWNTs $(\Theta=1)$ can be explained by the fact that the adsorption of $\mathrm{H}$ alternatively inside and outside leads to the atomic configuration closer to the diamond structure having rather large band gap $\left(E_{g}=5.4 \mathrm{eV}\right)$.

The effect of hydrogenation on the electronic structure is even more remarkable at $\Theta=0.5$ and is the most interesting aspect of our study. Depending on the pattern of hydrogen adsorption, an isomer can be either a metal or insulator. For example, all uniform $(n, 0) \mathrm{H}-\mathrm{SWNT}$ are metallic. On the other hand, the chain pattern realized on the $(n, 0)$ SWNTs results in two doubly degenerate, almost dispersionless states at the valence and conduction band edges. The band gap $E_{g}$ between these states decreases with increasing $R$. When $n$ is odd, $E_{g}$ is large [e.g $E_{g}=2.1 \mathrm{eV}$ for $\left.(7,0)\right]$. When $n$ is even, the doubly degenerate band at the conduction band edge moves towards the valence band edge and splits as bonding and antibonding states. As a result $E_{g}$ is reduced significantly becoming only a pseudogap for large and even $n$. Finally, the dimer row isomers are insulators and the $E_{g}$ increases with increasing radius. Surprisingly, there are two dispersive bands with $\sim 1 \mathrm{eV}$ bandwidth at both band edges and extremum moves from the center of Brillioun zone ( $\Gamma$ point) to the zone edge ( $Z$ point).

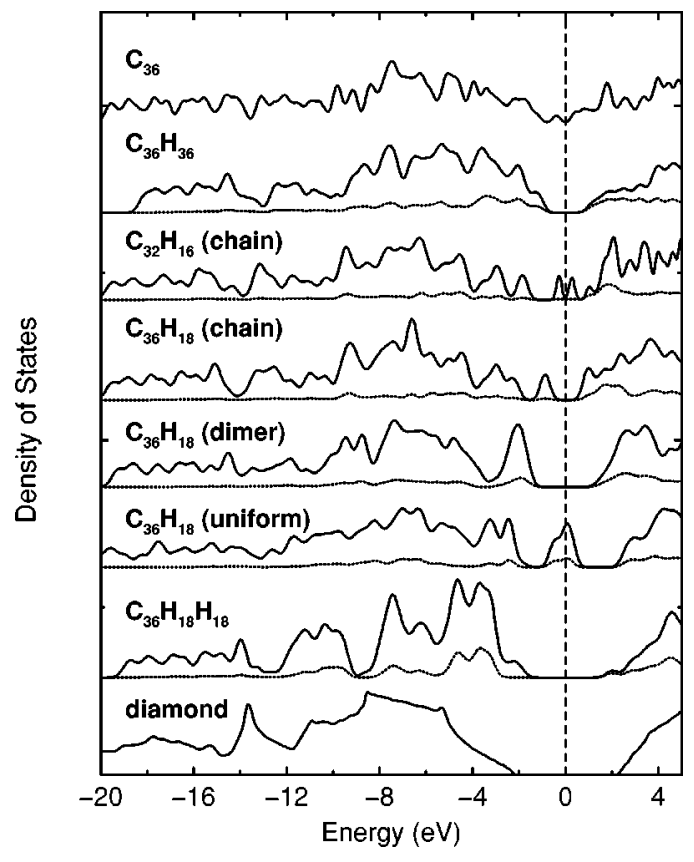

FIG. 4. Comparison of the electronic density of states (DOS) of a bare $(9,0)$ nanotube $\left(\mathrm{C}_{36}\right)$ and its various hydrogenated isomers. DOS of a chain pattern $(8,0) \mathrm{H}-\mathrm{SWNT}$ at half coverage $\left(\mathrm{C}_{32} \mathrm{H}_{16}\right)$ and bulk diamond (bottom panel) are also shown for comparison. The zero of energy is taken at the Fermi energy showed by vertical dashed line. The dotted lines are the partial density of states resolved on hydrogen atoms.

Hydrogen adsorption induced dramatic changes of electronic structure are demonstrated by total density of states (DOS), $\mathcal{D}(E)$, of $(9,0)$ nanotubes in Fig. 4. First of all, the small band gap of the bare $(9,0)$ SWNT is opened by $\sim 2 \mathrm{eV}$ upon exohydrogenation at $\Theta=1$. The band gap is still significant for $\Theta=0.5$ with the chain pattern, and increases to 4 $\mathrm{eV}$ for dimer pattern. However, a similar chain pattern in the $(8,0) \mathrm{H}-\mathrm{SWNT}$ (see $\mathrm{C}_{32} \mathrm{H}_{16}$ in Fig. 4) has a much smaller band gap. Suprisingly, all zigzag nanotubes uniformly exohydrogenated at $\Theta=0.5$ are metals. As displayed in the sixth panel of Fig. 4 for $\mathrm{C}_{36} \mathrm{H}_{18}$ (uniform), their total density of states are characterized by a peak yielding high state density at $E_{F}$. While carbon states are pushed apart, yielding a $\sim 4-5 \mathrm{eV}$ gap, a new dispersive metallic band with $\sim 1-2 \mathrm{eV}$ bandwidth crosses the Fermi level. Apart from being an ideal 1D conductor, this very high density of states at $E_{F}$ might lead to superconductivity. Note that, these uniform $\mathrm{H}-\mathrm{SWNT}$ undergo a massive reconstruction and their circular cross sections change into square ones with the formation of new $\mathrm{C}-\mathrm{C}$ bonds $\left(\mathrm{C}_{4}\right)$ at the corners. All $\mathrm{C}$ atoms without $\mathrm{H}$ attached (except those at corners) as well as the $\mathrm{H}$ atoms at the center of four planar sides contribute to the high $\mathcal{D}\left(E_{F}\right)$. This way four individual conduction paths are formed on each side of square tube. It is emphasized that the transformation from the $s p^{2}$ to the $s p^{3}$ bonding underlies various effects discussed in this study. The H-SWNTs, especially $\mathrm{C}_{4 n} \mathrm{H}_{2 n} \mathrm{H}_{2 n}$ structures can be conceived as if they are more diamondlike than graphitic. Our arguments are justified by the comparison of $\mathcal{D}(E)$ of the endo-exohydrogenated 
$(9,0)$ with that of bulk diamond in Fig. 4. Apart from opening a large band gap, the quasimetallic $\mathcal{D}(E)$ of the bare $(9,0)$ is modified to become similar to that bulk diamond. The latter has relatively larger valence band width due to coupling of distant neighbors.

In conclusion, our study reveals many important and novel effects of hydrogen adsorption on SWNTs, and brings a number of new problems and issues to be explored. For example, one can argue that the band gap of a SWNT can be engineered by the controlled hydrogenation of a single nanotube as in the alloy of $\mathrm{Si}_{x} \mathrm{Ge}_{1-x}$. A number of isomers which can be tailored with different hydrogen decoration provide options in developing new materials. Furthermore, multiple quantum well structures, or one-dimensional chain of quantum dots, can be tailored by periodic and modulated hydrogenation of a single nanotube. Finally, the very high density of states at the Fermi level of uniform pattern isomer at half coverage may result in to superconductivity in SWNT based nanowires. Needless to say, realization of the systems proposed here will be an experimental challenge. However, the fact that other carbon clusters such as cubane, dodecahedrane, and $\mathrm{C}_{60} \mathrm{H}_{32}$ have been successfully synthesized suggests that this is not impossible.

This work was partially supported by the NSF under Grant No. INT01-15021 and TUBITAK under Grant No. TBAG-U/13(101T010).
${ }^{1}$ S. Iijima, Nature (London) 354, 56 (1991).

${ }^{2}$ R. Saito, G. Dresselhaus, and M.S. Dresselhaus, Physical Properties of Carbon Nanotubes (Imperial College Press, London, 1998).

${ }^{3}$ N. Hamada, S. Sawada, and A. Oshiyama, Phys. Rev. Lett. 68, 1579 (1992).

${ }^{4}$ C.T. White, D.H. Robertson, and J.W. Mintmire, Phys. Rev. B 47, 5485 (1993).

${ }^{5}$ J.W.G. Wildöer et al., Nature (London) 391, 59 (1998); T.W. Odom et al., Nature (London) 391, 62 (1998).

${ }^{6}$ Z. Yao, C.L. Kane, and C. Dekker, Phys. Rev. Lett. 84, 4613 (2000).

${ }^{7}$ O. Gülseren, T. Yildirim, and S. Ciraci, Phys. Rev. B 65, 153405 (2002).

${ }^{8}$ A.C. Dillon et al., Nature (London) 386, 377 (1997); C. Liu et al., Science 286, 1127 (1999).

${ }^{9}$ K.N. Kudin, G.E. Scuseria, and B.I. Yakobson, Phys. Rev. B 64, 235406 (2001).

${ }^{10}$ K. Tada, S. Furuya, and K. Watanabe, Phys. Rev. B 63, 155405 (2001).
${ }^{11}$ Y. Ma, Y. Xia, M. Zhao, R. Wang, and L. Mei, Phys. Rev. B 63, 115422 (2001).

${ }^{12}$ S.M. Lee and Y.H. Lee, Appl. Phys. Lett. 76, 2877 (2000).

${ }^{13}$ S. Chan et al., Phys. Rev. Lett. 87, 205502 (2001).

${ }^{14}$ K.N. Kudin, H.F. Bettinger, and G.E. Scuseria, Phys. Rev. B 63, 045413 (2001).

${ }^{15}$ A.N. Andriotis, M. Menon, D. Srivastava, and G. Froudakis, Phys. Rev. B 64, 193401 (2001).

${ }^{16}$ T. Yildirim, O. Gülseren, and S. Ciraci, Phys. Rev. B 64, 075404 (2001).

${ }^{17}$ O. Gülseren, T. Yildirim, and S. Ciraci, Phys. Rev. Lett. 87, 116802 (2001).

${ }^{18}$ D. Srivastava et al., J. Phys. Chem. B 103, 4330 (1999).

${ }^{19}$ P.G. Collins et al., Science 287, 1801 (2000); J. Kong et al., ibid. 287, 622 (2000).

${ }^{20}$ J.P. Perdew and Y. Wang, Phys. Rev. B 46, 6671 (1992).

${ }^{21}$ M.C. Payne et al., Rev. Mod. Phys. 64, 1045 (1992).

${ }^{22}$ X. Blase, L.X. Benedict, E.L. Shirley, and S.G. Louie, Phys. Rev. Lett. 72, 1878 (1994). 